\title{
An Anionic Polymer Incorporating Low Amounts of Hydrophobic Residues Is a Multifunctional Surfactant. Part 1: Emulsifying, Thickening, Moisture-Absorption and Moisture-Retention Abilities of a Fatty Acid-Containing Anionic Polysaccharide
}

\author{
Makoto Urai ${ }^{12 *}$, Tomoko Aizawa1, Mutsuyasu Nakajima1, Michio Sunairi1 \\ ${ }^{1}$ Laboratory of Molecular Microbiology, Department of Applied Biological Science, College of Bioresource \\ Sciences, Nihon University, Fujisawa, Japan \\ ${ }^{2}$ Department of Chemotherapy and Mycoses, National Institute of Infectious Diseases, Tokyo, Japan \\ Email: urai@niid.go.jp
}

Received 25 February 2015; accepted 26 March 2015; published 1 April 2015

Copyright (C) 2015 by authors and Scientific Research Publishing Inc.

This work is licensed under the Creative Commons Attribution International License (CC BY).

http://creativecommons.org/licenses/by/4.0/

(c) (i) Open Access

\section{Abstract}

We have been studying the function and structure of fatty acid-containing extracellular polysaccharides (FACEPS) produced by bacteria belonging to the genus Rhodococcus. In this study, we examined the relationships between the structure and emulsifying, thickening, moisture-absorption, and moisture-retention capabilities of rhodococcal FACEPS using S-2 EPS produced by $R$. rhodochrous strain S-2. We prepared chemically deacylated S-2 EPS (DeAcyl S-2 EPS) and palmitoylated DeAcyl S-2 EPS (ReAcyl S-2 EPS), and compared them with native S-2 EPS. All of the properties were attenuated and recovered by deacylation and reacylation of S-2 EPS, respectively. These results suggest that the fatty acid moiety of rhodococcal FACEPS is involved in such functional properties. We also showed that palmitoylation improved the emulsifying, moisture-absorption, and moisture-retention abilities of other acidic polysaccharides that are commercially available. These results suggest that the acidity of the polysaccharide backbone is at least partly responsible for the observed functionality of fatty acid-containing polysaccharides. To our knowledge, this is the first report on multifunctional property of an anionic polymer incorporating low

\footnotetext{
"Corresponding author.
}

How to cite this paper: Urai, M., et al. (2015) An Anionic Polymer Incorporating Low Amounts of Hydrophobic Residues Is a Multifunctional Surfactant. Part 1: Emulsifying, Thickening, Moisture-Absorption and Moisture-Retention Abilities of a Fatty Acid-Containing Anionic Polysaccharide. Advances in Chemical Engineering and Science, 5, 173-180. 
amounts of hydrophobic residues. The present findings could be useful for the creation of new multifunctional surfactants from renewable raw materials for use in various industries, e.g., in cosmetics.

\title{
Keywords
}

\author{
Rhodococcus, Fatty Acid-Containing Extracellular Polysaccharide (FACEPS), Emulsification, \\ Thickening, Moisture Absorption, Moisture Retention
}

\section{Introduction}

We have been studying the function and structure of extracellular polysaccharides (EPS) produced by several Rhodococcus species [1]-[8], and we demonstrated the potential commercial application of an EPS, prepared from a mucoidal mutant, SM-1, of Rhodococcus rhodochrous ATCC 12674 (SM-1 EPS), as an emulsifier and in moisture-absorbent and moisture-retention materials [1]. SM-1 EPS was found to absorb and retain moisture in both dry and high-temperature environments at a much greater extent than known moisture absorbents or retentioners such as silica gel, glycerol, and hyaluronic acid. SM-1 EPS contains galactose, glucose, fucose, and glucuronic acid at a molar ratio of 6:3:2:4 together with 1.2\% (W/W) stearic acid, 2.3\% (W/W) palmitic acid, and $10.3 \%(\mathrm{~W} / \mathrm{W})$ pyruvic acid. We also reported the potential for commercial application of several rhodococcal EPSs as thickeners and emulsifiers in addition to its use in bioremediation to clean up oil-spills in marine environments [2]-[4]. These rhodococcal EPSs are high-molecular-weight $(>2,000,000)$ anionic polysaccharides containing fatty acids, mainly stearic acid and palmitic acid, via alkaline-labile bonds, probably ester bonds. We designated these EPS as fatty acid-containing extracellular polysaccharides (FACEPS).

Recently, we determined the sugar-chain structure of one of these rhodococcal FACEPS, the EPS produced by $R$. rhodochrous strain S-2 (S-2 EPS) [5]. S-2 EPS consists of a tetrasaccharide repeating unit with an $\rightarrow 3$ )- $\alpha$ D-mannose-( $1 \rightarrow 3)$ - $\alpha$-D-glucose- $(1 \rightarrow 3)-\alpha$-D-galactose-( $1 \rightarrow$ backbone with side chains of $\beta$-D-glucuronic acid residues bound to the C-2 position of the mannose residue via glycosyl bond, and it contains $0.8 \%(\mathrm{~W} / \mathrm{W})$ stearic acid and 2.7\% (W/W) palmitic acid. However, the relationships between the structure and emulsifying, thickening, moisture-absorption and retention properties of the rhodococcal FACEPS are unclear.

In these two accompanying papers, we address the multifunctional properties of an anionic polymer incorporating a low amount of hydrophobic residues, in particular a fatty acid-containing anionic polysaccharide (this paper, Part 1) and an alkyl-esterified poly- $\gamma$-glutamic acid (following paper, Part 2). In this paper, we examined the relationships between the structure and emulsifying, thickening, moisture-absorption and moisture-retention properties of the rhodococcal FACEPS using S-2 EPS, and we suggest that the fatty acid moieties of S-2 EPS underlie these. Furthermore, we also examined the effect of the polysaccharide backbone structure on these properties through the palmitoylation of various polysaccharides that are commercially available.

\section{Materials and Methods}

\subsection{Materials}

The S-2 EPS was prepared as described by Urai et al. [5]. Hyaluronic acid from cockscomb was purchased from Tokyo Kasei Kogyo Co. (Tokyo, Japan), and the molecular sieve, glycerol, silica gel, and urea were obtained from Wako Pure Chemical Industries, Ltd. (Osaka, Japan). Sumifloc FA-70 (anionic synthetic high-polymer absorbent: polyacrylamide derivative; MW $350 \times 10^{4}$ ) was purchased from MT Aquapolymer, Inc. (Tokyo, Japan). Alginic acid (Keltone, Kelco Co., Atlanta, GA, USA), carboxymethyl cellulose (Cellogen BS, Dai-Ichi Kogyo Seiyaku Co., Ltd., Kyoto, Japan), and dextran T2000 (Amersham Biosciences Corp., Piscataway, NJ, USA) were also purchased.

\subsection{Deacylation of S-2 EPS}

Purified S-2 EPS was suspended in $1 \mathrm{M}$ sodium hydroxide aqueous solution and heated at $100^{\circ} \mathrm{C}$ for $1 \mathrm{~h}$. After cooling, it was neutralized with aqueous $\mathrm{HCl}$, dialyzed against MilliQ water, and then lyophilized. The resulting deacylated EPS was analyzed by NMR and GC-MS to confirm deacylation. 


\subsection{Palmitoylation of Polysaccharides}

Palmitoylation reactions were conducted as described by Tian et al. with some modifications [9]. Substrates (ca. $200 \mathrm{mg}$ ) were soaked in pyridine $\left(2 \mathrm{ml}\right.$ ) for $16 \mathrm{~h}$ at $80^{\circ} \mathrm{C}$, and then pyridine was removed by drying in vacuo. Dried substrates were immersed in mixtures of $1.7 \mathrm{ml}$ of toluene and $0.2 \mathrm{ml}$ of pyridine and heated at $80^{\circ} \mathrm{C}$ for $12 \mathrm{hr}$. After the mixtures were cooled in an ice-salt bath for $30 \mathrm{~min}$, aliquots $(0.12 \mathrm{ml})$ of palmitoyl chloride (Wako Pure Chemical Industries Co. Ltd., Kyoto, Japan) were slowly added to the reaction solutions with constant stirring. The solutions were cooled in an ice-salt bath for further $30 \mathrm{~min}$, and then heated at $80^{\circ} \mathrm{C}$ for $3.5 \mathrm{~h}$. After addition of 50\% acetone in MilliQ water (v/v) to each reaction vessel, the reaction solutions were centrifuged at $1000 \mathrm{~g}$ for $20 \mathrm{~min}$. Precipitates were washed with 50\% acetone in MilliQ water (v/v) again, and then in acetone once. The precipitates were immersed in MilliQ water at $4^{\circ} \mathrm{C}$ overnight, and water-soluble palmitoylated polysaccharides was recovered as supernatant by centrifugation at $1000 \times \mathrm{g}$ for $20 \mathrm{~min}$. The content of palmitic acid in the palmitoylated polysaccharides was determined by GC-MS after alkali hydrolysis.

\subsection{Emulsifying Ability}

The polysaccharide was dissolved in $10 \mathrm{ml}$ of MilliQ water with $100 \mathrm{mg}$ of Arabian light crude oil or Louisiana sweet crude oil in a glass vial, and shaken at $110 \mathrm{rpm}$ at $28^{\circ} \mathrm{C}$ for 16 hour. Emulsification was identified visually.

\subsection{Thickening Ability}

The viscosity of the native and modified S-2 EPS was determined as described previously [2].

\subsection{Moisture Absorption and Retention Capacities}

The moisture absorption and retention capacities were measured as described by Urai et al. [1]. Briefly, constant relative humidity conditions were established at $37^{\circ} \mathrm{C}$. Lyophilized samples were dried at $105^{\circ} \mathrm{C}$ overnight, placed in glass weighing vessels and weighed. For determination of the moisture absorption capacity, the samples in the vessels were placed in desiccators for $24 \mathrm{~h}$ and then weighed again. The moisture absorption capacity was calculated as follows:

$$
\begin{aligned}
& \text { Moisture absorption capacity }(\%) \\
& =\{(\text { sample weight after } 24 \mathrm{~h}-\text { initial sample weight }) /(\text { initial sample weight })\} \times 100
\end{aligned}
$$

For measurement of the moisture retention capacity, the vessels containing the dried samples were weighed again after addition of MilliQ water (20\% of sample weight) with soaking. The vessels were left for $24 \mathrm{~h}$ in the desiccators and then weighed. The moisture retention capacity was calculated as follows:

Moisture retention capacity (\%)

$=\{1-($ initial sample weight-sample weight after $24 \mathrm{~h}) /($ weight of the added water $)\} \times 100$

\section{Results and Discussion}

\subsection{Chemical Modification of S-2 EPS}

The native S-2 EPS contains 0.8\% (W/W) stearic acid and 2.7\% (W/W) palmitic acid as described previously [5]. To elucidate the functions of the fatty acid residues of S-2 EPS, the residues were removed by mild alkali hydrolysis (deacylated S-2 EPS, DeAcyl S-2 EPS), and the deacylated S-2 EPS was reacylated by palmitoylation (ReAcyl S-2 EPS). The S-2 EPS was successfully deacylated by mild alkali hydrolysis, as fatty acids were not detected in DeAcyl S-2 EPS by GC-MS and NMR analysis. Palmitoylation was then performed for this DeAcyl S-2 EPS. The palmitic acid content of the ReAcyl S-2 EPS was 1.7\% (W/W). DeAcyl S-2 EPS was successfully reacylated with similar amounts of fatty acid to the native S-2 EPS.

\subsection{Effects of Fatty Acid Residues on the Emulsifying Ability of S-2 EPS}

The emulsifying ability of native S-2 EPS and chemically modified S-2 EPSs was determined visually after shaking with crude oil. The native S-2 EPS showed good emulsifying ability for crude oil in MilliQ water 
(Figure 1). This ability was completely lost after deacylation, and recovered in ReAcyl S-2 EPS. These results suggest that the fatty acid moieties of S-2 EPS are important for its emulsifying property.

\subsection{Effects of Fatty Acid Residues on the Thickening Ability of S-2 EPS}

The viscosity of the native S-2 EPS was $4.68 \mathrm{dl} / \mathrm{g}$ at $27^{\circ} \mathrm{C}$, which was higher than the $3.90 \mathrm{dl} / \mathrm{g}$ for carboxymethyl cellulose (CM-cellulose), suggesting the possibility of its application as a thickener. In contrast, the viscosity of the deacylated S-2EPS was attenuated to $2.73 \mathrm{dl} / \mathrm{g}$, which suggests that the fatty acid moieties of S-2 EPS are involved in its thickening property.

\subsection{Characterization of Moisture Retention and Absorption Capacities of S-2 EPS}

S-2 EPS retained $79 \%$ of its initial moisture (which was equivalent to $16 \%$ of the weight of EPS) after $24 \mathrm{~h}$ under dry $(11 \%$ relative humidity, $\mathrm{RH})$ and high-temperature $\left(37^{\circ} \mathrm{C}\right)$ conditions (Table 1$)$. Its moisture retention capacity was much higher than that of commercially available moisture retainers such as hyaluronic acid or glycerol, and slightly higher than that of SM-1 EPS. Under 32\% RH, the S-2 EPS showed increased weight, suggesting that the EPS not only retains its initial moisture but also absorbs moisture from dry and high-temperature atmospheres. The S-2 EPS absorbed more than $19 \%$ and $8 \%$ of its initial weight in moisture under dry (32\% and $11 \% / \mathrm{RH}$, respectively) and high-temperature $\left(37^{\circ} \mathrm{C}\right)$ conditions (Table 2$)$. This absorption capability was much lower than that of SM-1 EPS but similar to that of other moisture absorbents tested, such as silica gel, glycerol, and hyaluronic acid. These data indicate that S-2 EPS possesses favorable moisture retention and moisture absorption properties.

Table 1. Moisture retention capacity of S-2 EPS and palmitoylated polysaccharides.

\begin{tabular}{|c|c|c|}
\hline & \multicolumn{2}{|c|}{ Moisture retention capacity (\%) } \\
\hline & Relative humidity $32 \%^{\mathrm{a}}$ & Relative humidity $11 \%^{\mathrm{a}}$ \\
\hline S-2 EPS & $>100^{\mathrm{b}}$ & $79 \pm 12$ \\
\hline DeAcyl S-2 EPS & $22 \pm 9$ & $4 \pm 6$ \\
\hline ReAcyl S-2 EPS & $>100^{\mathrm{b}}$ & $>100^{\mathrm{b}}$ \\
\hline SM-1 EPS ${ }^{d}$ & $>100^{\mathrm{b}}$ & $57 \pm 17$ \\
\hline Hyaluronic acid ${ }^{\mathrm{d}}$ & $100 \pm 24$ & $36 \pm 7$ \\
\hline Urea $^{\mathrm{d}}$ & $0 \pm 0$ & $\mathrm{NT}^{\mathrm{c}}$ \\
\hline Glycerol $^{\mathrm{d}}$ & $52 \pm 5$ & $8 \pm 5$ \\
\hline Silica gel $^{\mathrm{d}}$ & $76 \pm 1$ & $27 \pm 6$ \\
\hline Molecular sieve & $54 \pm 11$ & $68 \pm 9$ \\
\hline Sumifloc FA-70 & $17 \pm 10$ & $6 \pm 5$ \\
\hline CM-cellulose & $50 \pm 5$ & $49 \pm 7$ \\
\hline Palmitoylated CM-cellulose & $84 \pm 10$ & $84 \pm 6$ \\
\hline Alginic acid & $87 \pm 16$ & $49 \pm 17$ \\
\hline Palmitoylated alginic acid & $64 \pm 32$ & $37 \pm 7$ \\
\hline Dextran T2000 & $65 \pm 11$ & $27 \pm 11$ \\
\hline Palmitoylated dextran T2000 & $45 \pm 16$ & $12 \pm 3$ \\
\hline
\end{tabular}

${ }^{\mathrm{a}}$ Values were calculated according to the equation described in the Materials and Methods; ${ }^{\mathrm{b}}$ The weights of samples were greater than their initial weights; ${ }^{\mathrm{C}} \mathrm{NT}$, not tested; ${ }^{\mathrm{d}}$ These data are from a previous paper [1]. 


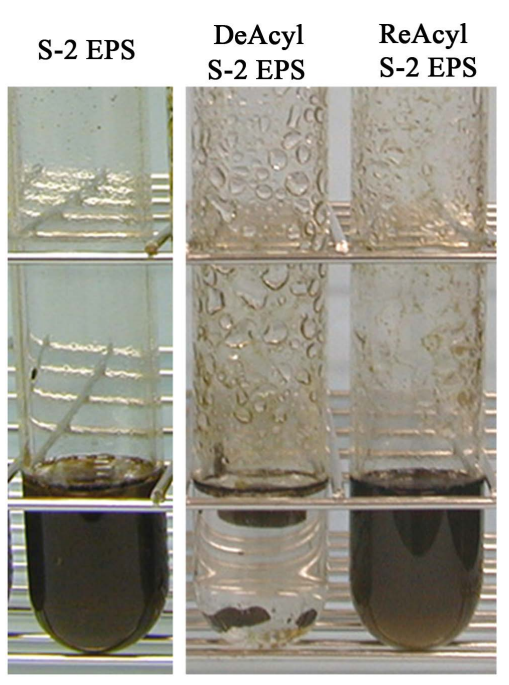

Figure 1. Effect of fatty acid residues on the emulsifying property of S-2 EPS. The final concentration of the EPSs was $0.1 \mathrm{mg} / \mathrm{ml}$, and that of Arabian light crude oil was $10 \mathrm{mg} / \mathrm{ml}$.

Table 2. Moisture absorption capacity of S-2 EPS and palmitoylated polysaccharides.

\begin{tabular}{|c|c|c|}
\hline & \multicolumn{2}{|c|}{ Moisture absorption capacity (\%) } \\
\hline & Relative humidity $32 \%^{\mathrm{a}}$ & Relative humidity $11 \%^{\mathrm{a}}$ \\
\hline S-2 EPS & $19 \pm 3$ & $8 \pm 1$ \\
\hline DeAcyl S-2 EPS & $10 \pm 4$ & $7 \pm 6$ \\
\hline ReAcyl S-2 EPS & $28 \pm 4$ & $19 \pm 4$ \\
\hline SM-1 EPS & $120 \pm 2$ & $17 \pm 12$ \\
\hline Hyaluronic acid ${ }^{\mathrm{c}}$ & $37 \pm 13$ & $11 \pm 1$ \\
\hline Urea $^{c}$ & $0 \pm 0$ & $\mathrm{NT}^{\mathrm{b}}$ \\
\hline Glycerol $^{\mathrm{c}}$ & $8 \pm 0$ & $3 \pm 1$ \\
\hline Silica gel ${ }^{\mathrm{c}}$ & $17 \pm 3$ & $6 \pm 1$ \\
\hline Molecular sieve & $10 \pm 1$ & $13 \pm 1$ \\
\hline Sumifloc FA-70 & $1 \pm 2$ & $0 \pm 0$ \\
\hline CM-cellulose & $11 \pm 4$ & $10 \pm 1$ \\
\hline Palmitoylated CM-cellulose & $19 \pm 4$ & $21 \pm 4$ \\
\hline Alginic acid & $16 \pm 4$ & $7 \pm 3$ \\
\hline Palmitoylated alginic acid & $13 \pm 5$ & $6 \pm 1$ \\
\hline Dextran T2000 & $12 \pm 2$ & $6 \pm 1$ \\
\hline Palmitoylated dextran T2000 & $10 \pm 3$ & $4 \pm 3$ \\
\hline
\end{tabular}

${ }^{a}$ Values were calculated according to the equation described in the Materials and Methods; ${ }^{b} \mathrm{NT}$, not tested; ${ }^{\mathrm{c}}$ These data are from a previous paper [1].

\subsection{Effects of Fatty Acid Residues on the Moisture Retention and Moisture Absorption Capacities of S-2 EPS}

DeAcyl S-2 EPS retained far less moisture than the native S-2 EPS (Table 1), and ReAcyl S-2 EPS showed recovery of the moisture retention property, and even under dry $(11 \% \mathrm{RH})$ and high-temperature $\left(37^{\circ} \mathrm{C}\right)$ conditions, the ReAcyl S-2 EPS showed increased weight, indicating that the EPS not only retains its initial moisture 
but also absorbs moisture from dry and high-temperature atmospheres. Similarly, the moisture absorption capacity of S-2 EPS was decreased by deacylation, and that of the DeAcyl S-2 EPS was restored or improved by palmitoylation (Table 2). These data suggest that the fatty acid moieties of S-2 EPS play important roles in the moisture retention and moisture absorption properties of the EPS.

Several reports have indicated moisture-retention or moisture-absorption abilities for some polysaccharides. An acidic polysaccharide containing fucose, glucose, rhamnose, and glucuronic acid produced by Alcaligenes latus B-16 is commercially available as a bioabsorbant [10] [11]. Low et al. showed that the starter strain Streptococcus thermophilus MR-1C produces a capsular polysaccharide responsible for the water-binding properties of MR-1C in cheese, and the $S$. thermophilus MR-1C EPS has a novel basic repeating unit composed of D-galactose, L-rhamnose, and L-fucose in a ratio of 5:2:1 [12]. These polysaccharides possess hydrophobic groups such as methyl glycose in their chemical structures. Hyaluronic acid is widely used in cosmetics as a humectant, and it is an acidic polysaccharide containing acetyl moieties as hydrophobic groups. Lingyum et al. reported that the moisture absorption and moisture retention abilities of carboxymethyl chitosan (CM-chitosan) are dependent on the degree of acetylation [13]. These polysaccharides typically possess hydrophobic groups in their structure, but their polysaccharide backbone structure varies. We therefore examined the effect of the polysaccharide backbone structure on the emulsifying, moisture retention, and moisture absorption properties by palmitoylating various commercially available polysaccharides.

\subsection{Effect of Palmitoylation on Emulsifying, Moisture Retention, and Moisture Absorption Properties of Commercially Available Polysaccharides}

CM-cellulose, alginic acid, and dextran were palmitoylated, and the palmitic acid content was determined by GC-MS as $0.3 \%, 0.9 \%$, and $0.2 \%(\mathrm{~W} / \mathrm{W})$, respectively. Figure 2 shows the emulsifying property of the palmitoylated polysaccharides. Palmitoylated alginic acid and CM-cellulose showed effective emulsification of crude oil, but palmitoylated dextran did not. These polysaccharides could not emulsify crude oil before palmitoylation (data not shown). Because alginic acid and CM-cellulose are acidic and dextran is a neutral polysaccharide, the acidic behavior of the polysaccharide backbone is important to for the emulsification mediated by palmitoylation.

The moisture retention and absorption properties of palmitoylated polysaccharides were also tested (Table 1 and Table 2, respectively). After palmitoylation, these properties were improved by approximately 1.7 fold in CM-cellulose, whereas the other polysaccharides showed a reduction rather than an improvement. These data suggest that the chemical structure of the sugar chain also affects the moisture retention and moisture absorption properties of the polysaccharide. The moisture retention and absorption properties of the Deacyl S-2 EPS and CM-cellulose, but not those of alginic acid and dextran, were improved by palmitoylation. Deacyl S-2 EPS and CM-cellulose share structural similarity with regard to acidity. Deacyl S-2 EPS contains one carboxyl group for every four glycose residues, and the CM-cellulose used in this study contains one carboxyl group for every two glucose residues. In contrast, alginic acid is a highly acidic polymer that contains a carboxyl group for every glycose residue. Dextran is a neutral homopolysaccharide consisting of glucose. Lingyum et al. described that
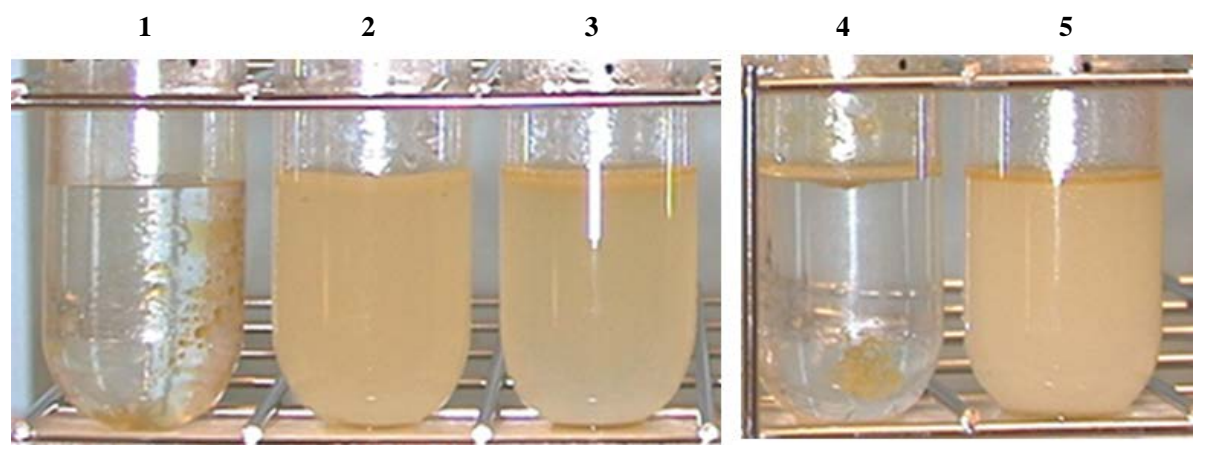

Figure 2. Effect of palmitoylation on the emulsifying property of commercially available polysaccharides. The final concentration of the polysaccharide was $0.5 \mathrm{mg} / \mathrm{ml}$, and that of Louisiana sweet crude oil was $10 \mathrm{mg} / \mathrm{ml}$. 1, No addition of polysaccharide; 2, palmitoylated alginic acid; 3, palmitoylated CM-cellulose; 4, palmitoylated dextran; and 5, S-2 EPS. 
the degree of carboxymethylation affects the moisture absorption and moisture retention abilities of the CMchitosan [13], and they suggested that carboxyl groups are important for the ability of CM-chitosan to absorb and retain moisture. However, a high degree of carboxymethylation causes strong hydrogen bonding between CM-chitosan chains, which prevents the adsorption and retention of moisture. In the case of the palmitoylated polysaccharide, the acidity of the polysaccharide backbone may be similarly involved in the moisture absorption and retention properties.

\section{Conclusion}

We showed that the fatty acid moiety of the rhodococcal FACEPS may be involved in the emulsifying, thickening, moisture-absorption and moisture-retention properties of the FACEPS. We also showed that the acidity of the polysaccharide backbone may play a role in these properties. To our knowledge, this is the first report of the multifunctional properties of an anionic polymer incorporating a low amount of hydrophobic residues. These observations are useful to create new multifunctional surfactants from renewable raw material with the potential to be used in various industries, e.g., cosmetics. To determine how the hydrophobic groups incorporated into the anionic polymer contribute to the high capacity of emulsification, moisture absorption and moisture retention, it is necessary to synthesize a polymer having a more simplified structure than polysaccharides. In the following paper (Part 2), we synthesized alkyl-esterified poly- $\gamma$-glutamic acid with various lengths of alkyl chains and degrees of substitution to examine the effect of the hydrophobic groups on the emulsifying, moisture absorption, and moisture retention properties of an anionic polymer.

\section{Acknowledgements}

We are grateful to T. Beppu for giving us the opportunity to complete this research. We thank K. Ueda at the Institute of Applied Life Science for allowing us to use its instrumental facilities. We also thank T. Fukuhara for his valuable discussion. We acknowledge H. Anzai and various members of our laboratories for their suggestions, encouragement, and technical assistance. This study was supported in part by grants from the Takano Life Science Research Foundation (Japan).

\section{References}

[1] Urai, M., Anzai, H., Iwabuchi, N., Sunairi, M. and Nakajima, M. (2002) A Novel Moisture-Absorbing Extracellular Polysaccharide from Rhodococcus rhodochrous SM-1. Actinomycetologica, 16, 26-31. http://dx.doi.org/10.3209/saj.16_26

[2] Urai, M., Anzai, H., Iwabuchi, N., Sunairi, M. and Nakajima, M. (2004) A Novel Viscous Extracellular Polysaccharide Containing Fatty Acids from Rhodococcus rhodochrous ATCC 53968. Actinomycetologica, 18, 15-17. http://dx.doi.org/10.3209/saj.18_15

[3] Urai M., Yoshizaki, H., Anzai, H., Ogihara, J., Iwabuchi, N., Harayama, S., Sunairi, M. and Nakajima, M. (2007) Structural Analysis of an Acidic, Fatty Acid Ester-Bonded Extracellular Polysaccharide Produced by a PristaneAssimilating Marine Bacterium, Rhodococcus erythropolis PR4. Carbohydrate Research, 342, 933-942. http://dx.doi.org/10.1016/j.carres.2007.02.001

[4] Iwabuchi, N., Sunairi, M., Urai, M., Itoh C., Anzai, H., Nakajima, M. and Harayama, S. (2002) Extracellular Polysaccharides of Rhodococcus rhodochrous S-2 Stimulate the Degradation of Aromatic Components in Crude Oil by Indigenous Marine Bacteria. Applied and Environmental Microbiology, 68, 2337-2343. http://dx.doi.org/10.1128/AEM.68.5.2337-2343.2002

[5] Urai, M., Anzai, H., Ogihara, J., Iwabuchi, N., Harayama, S., Sunairi, M. and Nakajima, M. (2006) Structural Analysis of an Extracellular Polysaccharide Produced by Rhodococcus rhodochrous Strain S-2. Carbohydrate Research, 341, 766-775. http://dx.doi.org/10.1016/j.carres.2005.12.013

[6] Aizawa, T., Neilan, B., Couperwhite, I., Urai, M., Anzai, H., Iwabuchi, N., Nakajima, M. and Sunairi, M. (2005) Relationship between Extracellular Polysaccharide and Benzene Tolerance of Rhodococcus sp. 33. Actinomycetologica, 19, 1-6. http://dx.doi.org/10.3209/saj.19.1

[7] Urai, M., Aizawa, T., Anzai, H., Ogihara, J., Iwabuchi, N., Neilan. B., Couperwhite, I., Nakajima, M. and Sunairi, M. (2006) Structural Analysis of an Extracellular Polysaccharide Produced by a Benzene Tolerant Bacterium, Rhodococcus sp. 33. Carbohydrate Research, 341, 616-623. http://dx.doi.org/10.1016/j.carres.2006.01.010

[8] Urai, M., Yoshizaki, H., Anzai, H., Ogihara, J., Iwabuchi, N., Harayama, S., Sunairi, M. and Nakajima, M. (2007) Structural Analysis of Mucoidan, an Acidic Extracellular Polysaccharide Produced by a Pristane-Assimilating Marine 
Bacterium, Rhodococcus erythropolis PR4. Carbohydrate Research, 342, 927-932. http://dx.doi.org/10.1016/j.carres.2007.02.002

[9] Tian, B., Dong, C. and Chen, L. (1998) Preparation of Konjac Glucomannan Ester of Palmitic Acid and Its Emulsification. Journal of Applied Polymer Science, 67, 1035-1038. http://dx.doi.org/10.1002/(SICI)1097-4628(19980207)67:6<1035::AID-APP10>3.0.CO;2-\#

[10] Kurane, R. and Nohata, Y. (1994) Biopolymer from Alcaligenes latus. Part II. A New Water-Absorbing Polysaccharide from Alcaligenes latus. Bioscience, Biotechnology, and Biochemistry, 58, 235-238. http://dx.doi.org/10.1271/bbb.58.235

[11] Kurane, R. and Nohata, Y. (1995) Identification of Consistuent Sugers of Polysaccharide Bioabsorbent from Alcaligenes latus. Bioscience, Biotechnology, and Biochemistry, 59, 908-911. http://dx.doi.org/10.1271/bbb.59.908

[12] Low, D., Ahlgren, J.A., Horne, D., McMahon, D.J., Oberg, C.J. and Broadbent, J.R. (1998) Role of Streptococcus thermophilus MR-1C Capsular Exopolysaccharide in Cheese Moisture Retention. Applied and Environmental Microbiology, 64, 2147-2151.

[13] Lingyun, C., Yumin, D. and Xiaoqing, Z. (2003) Relationships between the Molecular Structure and MoistureAbsorption and Moisture-Retention Abilities of Carboxymethyl Chitosan II. Effect of Degree of Deacetylation and Carboxymethylation. Carbohydrate Research, 338, 333-340. http://dx.doi.org/10.1016/S0008-6215(02)00462-7 